\title{
A comprehensive study of shower to shower fluctuations
}

\author{
P.M. Hansen ${ }^{\text {a }}$, J. Alvarez-Muñiz ${ }^{\text {b,* }}$, R.A. Vázquez ${ }^{\text {b }}$ \\ a Departamento de Física, IFLP CONICET Facultad de Ciencias Exactas, Universidad Nacional de La Plata C.C. 67, 1900 La Plata, Argentina \\ ${ }^{\mathrm{b}}$ Departamento de Física de Partículas E' Instituto Galego de Física de Altas Enerxías (IGFAE), Facultade de Física, Universidade de Santiago de Compostela, \\ 15782 Santiago de Compostela, Spain
}

\section{A R T I C L E I N F O}

Article history:

Received 21 April 2010

Received in revised form 8 September 2010

Accepted 1 November 2010

Available online 4 November 2010

\section{Keywords:}

Cosmic rays

Extensive air showers

Ground detector

Simulation

Muon component

Electromagnetic component

\begin{abstract}
A B S T R A C T
By means of Monte Carlo simulations of extensive air showers (EAS), we have performed a comprehensive study of the shower to shower fluctuations affecting the longitudinal and lateral development of EAS. We split the fluctuations into physical fluctuations and those induced by the thinning procedure customarily applied to simulate showers at EeV energies and above. We study the influence of thinning on the calculation of the shower to shower fluctuations in the simulations. For thinning levels larger than $R_{\text {thin }}=10^{-5}-10^{-6}$, the determination of the shower to shower fluctuations is hampered by the artificial fluctuations induced by the thinning procedure. However, we show that shower to shower fluctuations can still be approximately estimated, and we provide expressions to calculate them. The influence of fluctuations of the depth of first interaction on the determination of shower to shower fluctuations is also addressed.
\end{abstract}

(ㄷ) 2010 Elsevier B.V. All rights reserved.

\section{Introduction}

Extensive air showers (EAS) have been studied over the last 70 years [1]. They result from the interaction in the atmosphere of high-energy protons and nuclei arriving from space. The product of these collisions are a set of secondary particles carrying a fraction of the primary energy. These secondaries move through the atmosphere and interact again generating new secondaries. The process continues, increasing the number of secondary particles, until their energies are too low to contribute to the generation of new particles. Particles reaching ground are sampled with arrays of detectors, and their properties are used to infer the properties of the primary initiating the shower. Measurements of the electron and muon density, of the arrival time of the particles at ground, and of the depth at which the shower has the maximum number of particles $\left(\mathrm{X}_{\max }\right)$, give information on the arrival direction, primary energy, and on the mass of the primaries [1].

The complexity of the cascade phenomena, and the poor knowledge of the hadronic interactions at very high energy [2], make the experimental determination of the properties of the primaries very difficult. Moreover, primary particles with the same energy, mass and direction produce secondary particles with parameters that vary from shower to shower. This feature is called "shower to shower fluctuations". An understanding of the shower to shower fluctuations will help to improve the interpretation of cosmic-ray data.

\footnotetext{
* Corresponding author. Tel.: +34 981563100; fax: +34 981521091.

E-mail address: jaime.alvarezmuniz@gmail.com (J. Alvarez-Muñiz).
}

The calculation of shower to shower fluctuations can in principle be addressed with Monte Carlo simulations of extensive air showers. However, the number of particles that are produced in an air shower at ultra high energy (above $\sim 10^{18} \mathrm{eV}$ ) is so large $\left(\sim 10^{10}\right)$, that it is almost impossible to follow the propagation to ground level of all the secondaries in the Monte Carlo in a reasonable amount of time, or even to store the large amount of information produced. For this reason, a statistical sampling procedure called "thinning" [3] is used in the simulations. Thinning algorithms typically consist on propagating only a small, representative fraction of the total number of particles in the shower, assigning statistical weights to the sampled particles to compensate for the rejected ones. However, thinning algorithms introduce artificial fluctuations in the simulated showers, hampering the determination of the intrinsic, physical shower to shower fluctuations with Monte Carlo simulations. For this reason the study of fluctuations using Monte Carlo simulations is quite difficult and uncertain. This is of utmost importance in cosmic-ray physics, since an incorrect assumption on the shower to shower fluctuations can lead to systematic errors on the determination of the parameters of the primary particles.

In this work we address the problem of determining the true, physical shower to shower fluctuations in Monte Carlo simulations, and quantify the effect of thinning on their determination. We give expressions that allow the estimation of physical fluctuations from Monte Carlo simulations, even in the case of relatively strongly thinned showers. Other recent approaches which study the effect of artificial fluctuations due to the thinning procedure are given in $[4,5]$ 
The paper is organized as follows: in Section 2 we describe the simulations performed in this work, and the thinning algorithm adopted. In Section 3 we identify the different sources of fluctuations in shower simulations. In Section 4 we perform a comprehensive study of the fluctuations in the longitudinal and lateral shower development, and give expressions that allow to separate physical shower to shower fluctuations from the artificial fluctuations induced by the thinning procedure, and we quantify for which thinning levels these expressions can be applied. In Section 5 we apply our results to study the behaviour of the physical fluctuations at ground. In Section 6, we quantify the influence on the shower to shower fluctuations of the depth of first interaction of the primary initiating the shower. Finally, we summarize our conclusions in Section 7. In the Appendix we give an explicit mathematical derivation of the expressions presented in Section 4.

\section{The simulations}

In this work we have used the air shower simulation program, AIRES [6,7], along with the hadronic model QGSJET01 [8] to simulate proton and iron-induced showers with primary energy $10^{19} \mathrm{eV}$. As explained above, due to the large number of particles that are created in the simulation, AIRES includes a statistical sampling algorithm, that consists on propagating a small, representative fraction of the total number of particles, assigning a statistical weight $w$ to the sampled particles to compensate for the rejected ones. The weight is adjusted in such a way that both the total energy and the average number of particles is guaranteed to be conserved.

Before the simulation starts, the user indicates, as an input to AIRES, the relative thinning level $R_{\text {thin }}$. The thinning energy $E_{\text {thin }}-$ the energy below which the thinning process starts - is defined as $E_{\text {thin }}=R_{\text {thin }} \times E_{p}$ where, $E_{p}$ is the primary energy. For ultra high energy cosmic ray shower simulations convenient values for the relative thinning are $R_{\text {thin }}=10^{-5}-10^{-9}$, but the actual choice depends on the purpose of the simulation. The thinning level affects both the simulation CPU time and the size of the output produced in the simulation, both typically behaving linearly with $R_{\text {thin }}^{-1}$. If we increase $R_{\text {thin }}$ by a factor of 10 the simulation speeds up by a similar factor, and the output is reduced accordingly, but the price to pay is an enhancement of the artificial fluctuations in the simulated showers as discussed below.

We describe here the thinning algorithm implemented in the AIRES code [7], originally due to Hillas [3]. At the beginning of the simulation, the primary particle is assigned a weight $w=1$. Then the primary is propagated and interacts in the atmosphere producing $n$ secondary particles. Before incorporating any secondary particle in the simulation, the energy of the primary $E_{p}$ which has generated that secondary is compared to $E_{\text {thin }}$. If $E_{p}>E_{\text {thin }}$, then all the secondaries with energy greater or equal than $E_{\text {thin }}$ are kept, and their weight is equal to the weight of the primary particle. Secondaries with energy less than $E_{\text {thin }}$ are kept with a probability $p_{i}=E_{i} / E_{\text {thin }}$ ( $E_{i}$ is the energy of $i$ th secondary), and their weight is adjusted so that $w_{i}=\left(1 / p_{i}\right) \times w$, with $w$ being the weight of the mother particle producing that secondary. On the other hand if $E_{p}<E_{\text {thin }}$, it means that the particle came from a previous thinning operation. Then, one and only one of all the produced secondaries say the $j$ th - is kept, with probability $p_{j}=E_{j} / \sum_{i=1}^{n} E_{i}$. Again, the weight of this particle is increased by a factor $w_{j}=\left(1 / p_{j}\right) \times w$.

To avoid confusion between particles and weights, we identify an entry with a particle explicitly followed in the simulation which is associated a weight $w$. Hence, an entry represents $w$ particles. It is important to stress that once the thinning energy is reached, the number of entries $N_{e}$ is no longer increased in the shower processes (only one secondary particle is followed in each interaction), while the number of particles $N$ does however increase, since the weight of each entry typically increases in the showering process. When evaluating a physical observable, each entry must be weighted with its corresponding statistical weight.

In AIRES, the thinning algorithm is complemented with an "extended thinning algorithm" [7], designed to ensure that all the statistical weights are always smaller than a certain positive number (other algorithms based on this same idea are possible, see for instance [9]). To ensure this, an external parameter called statistical weight factor $W$ is available in the simulation, which limits the maximum weight $w_{\max }$ of the particles. To further optimize the procedure of sampling, different weight factors for electromagnetic and heavy particles are defined. These translate into different maximum weights $w_{\max }$ for different particle species, which also depend on the primary energy and the thinning energy. For instance, for a primary energy of $10^{19} \mathrm{eV}$, and a thinning level $R_{\text {thin }}=10^{-6}$ the maximum weight for electrons is approximately $w_{\max } \sim 10^{6}$ while for muons $w_{\max } \sim 10^{4}$.

We have simulated proton and iron-induced showers with primary energy $E_{p}=10^{19} \mathrm{eV}$, zenith angle $\theta=0^{\circ}, 30^{\circ}, 45^{\circ}$ and $60^{\circ}$ and relative thinning $R_{\text {thin }}=10^{-5}, 10^{-6}, 10^{-7}$ and $10^{-8}$ (the two latter $R_{\text {thin }}$ for proton only). We have also simulated showers with relative thinning of $R_{\text {thin }}=10^{-7}$ but with weight factor for electrons $W(\mathrm{EM})=0.1$ which translates into a smaller maximum weight than the default value given above. Finally, we have also simulated two sets of proton and iron-induced showers at $\theta=0^{\circ}$, with fixed depth of first interaction, starting at the corresponding mean interaction depth for protons and iron at $10^{19} \mathrm{eV}$.

\section{Fluctuations in EAS}

In a real shower or in a simulation of an EAS, there are a number of different fluctuations that can occur. Rather generally, we can make a simple classification as shown in Table 1.

"Physical fluctuations" are those due to physical processes in the shower. Here we split them into those due to the first interaction, and those occurring in the secondary interactions, as is customary, and because it has recently been suggested that "universal" shower properties may emerge when considering only the fluctuations in the first interaction point [10]. Physical fluctuations occurring in the first interaction are further divided into those affecting the depth of the first interaction, and those that arise from fluctuations of multiplicity or inelasticity also in the first interaction.

In the case of real data, fluctuations are enlarged due to the detector response, and to the fact that the detector usually only samples a small fraction of the shower front. This "sampling fluctuation" is a statistically well known problem, and sampling fluctuations are rather well studied $[11,12]$. We will not consider them in this work. Also the detector response introduces an additional source of fluctuations, which are detector dependent, and will not be considered here.

\section{Table 1}

Classification of the fluctuations in a shower, arising from the physical processes in the shower and the measurement process, and those that appear only in shower simulations.

\begin{tabular}{cl}
\hline Physical fluctuations & - Depth of first interaction \\
& - Multiplicity, inelasticity, etc., in first interaction \\
& - Secondary interactions \\
Experimental & - Detector response \\
fluctuations & - Sampling fluctuations \\
Artificial fluctuations & - Thinning \\
& - Un-thinning \\
\hline
\end{tabular}


On the other hand, Monte Carlo simulated data is affected by artificial fluctuations due to the thinning and un-thinning (re-sampling) procedures. For the purposes of this work we do not need to consider the effect of fluctuations induced by the unthinning procedure [11].

\section{Fluctuations of the longitudinal and lateral shower development}

\subsection{Fluctuations of the longitudinal profile}

In Fig. 1 we show the average longitudinal profile of the number of electrons (left panels) and muons (right panels) $\bar{N}$, obtained in simulations of 100 proton-induced showers with $E_{p}=10^{19} \mathrm{eV}$, for thinning levels $R_{\text {thin }}=10^{-6}$ and $10^{-7}$ and $\theta=0^{\circ}, 60^{\circ}$. Also shown are the relative shower to shower fluctuations $\sigma / \bar{N}$ for electrons and muons. As it is well known [13], the relative fluctuation has a minimum close to the depth of shower maximum. Also and as it is apparent from the figures, the dependence of the relative fluctuation on the thinning is small, at least for depths close to the depth of maximum.

In Fig. 2 we show for the same showers in Fig. 1, the skewness and the kurtosis of the distribution of the number of particles $N$ at different depths. It is worth recalling that the skewness of the distribution of a variable $x$ is defined as

$\gamma_{3}=\frac{\left\langle(x-\bar{x})^{3}\right\rangle}{\sigma_{x}^{3}}$

where $\bar{x}\left(\sigma_{x}\right)$ is the average (standard deviation) of $x$. The kurtosis is defined as:

$\gamma_{4}=\frac{\left\langle(x-\bar{x})^{4}\right\rangle}{\sigma_{x}^{4}}-3$

where the " -3 " in the definition is a convention to make $\gamma_{4}=0$ for a Gaussian distribution. Both the kurtosis and the skewness can be positive or negative. The skewness is a measure of the asymmetry of the distribution with respect to the mean value. A negative sign implies that the distribution is "deformed" towards values of $x$ smaller than the mean. The contrary applies for a positive sign.
The kurtosis is a measure of the length of the tails of the distribution. Positive values imply that the distribution has tails longer than those of a Gaussian, while negative values imply that the tails are shorter (for instance a flat distribution, a box, has kurtosis -1.2). A Gaussian distribution has $\gamma_{3}=\gamma_{4}=0$.

Several remarks can be made from Fig. 2. Firstly, it is apparent that both, the skewness and the kurtosis of the distribution of the number of particles depend strongly on the thinning level, contrary to what happens to the mean $\bar{N}$ and to the relative fluctuations $\sigma / \bar{N}$. Close to the depth of shower maximum both the skewness and the kurtosis have local extrema very different from zero, implying that the distribution is strongly non-Gaussian. The skewness is negative and this implies that the distribution is asymmetric towards smaller values of $N$ than average. The positive values of the kurtosis imply that the distribution of $N$ has tails longer than those of a Gaussian, at least close to shower maximum. Remarkably, the log-Gaussian distribution, widely used to parameterize fluctuations in the number of electrons, has both $\gamma_{3}>0$ and $\gamma_{4}>0$, while the fluctuations predicted by Monte Carlo simulations near the maximum of the shower, have negative skewness. For muons and at large depths the skewness is close to zero, so that a Gaussian or a log-Gaussian distribution is a good approximation. For electrons, this is never the case.

\subsection{Fluctuations of the lateral profile at ground}

Of special importance for cosmic-ray physics performed with arrays of detectors is the study of fluctuations in the number of particles at ground.

In Fig. 3 we show the relative fluctuations $(\sigma / \bar{N})$ of the total number of electrons (left panels) and muons (right panels) at ground (upper panels), and in a ring of width $\Delta r$ at a distance $r=1000 \mathrm{~m}$ from the shower axis (lower panels). In both cases the fluctuations are shown as a function of the number of showers simulated. The ring was taken from $r_{\min }=912 \mathrm{~m}$ to $r_{\max }=1092 \mathrm{~m}$, i.e. $\Delta r=180 \mathrm{~m}$ corresponding to a symmetric interval in the logarithm of $r$ around $r=1000 \mathrm{~m}$, chosen so that it compensates the decreasing density of particles with a larger area as $r$ increases. As expected, fluctuations in the ring $\Delta r$ are larger than the fluctuations
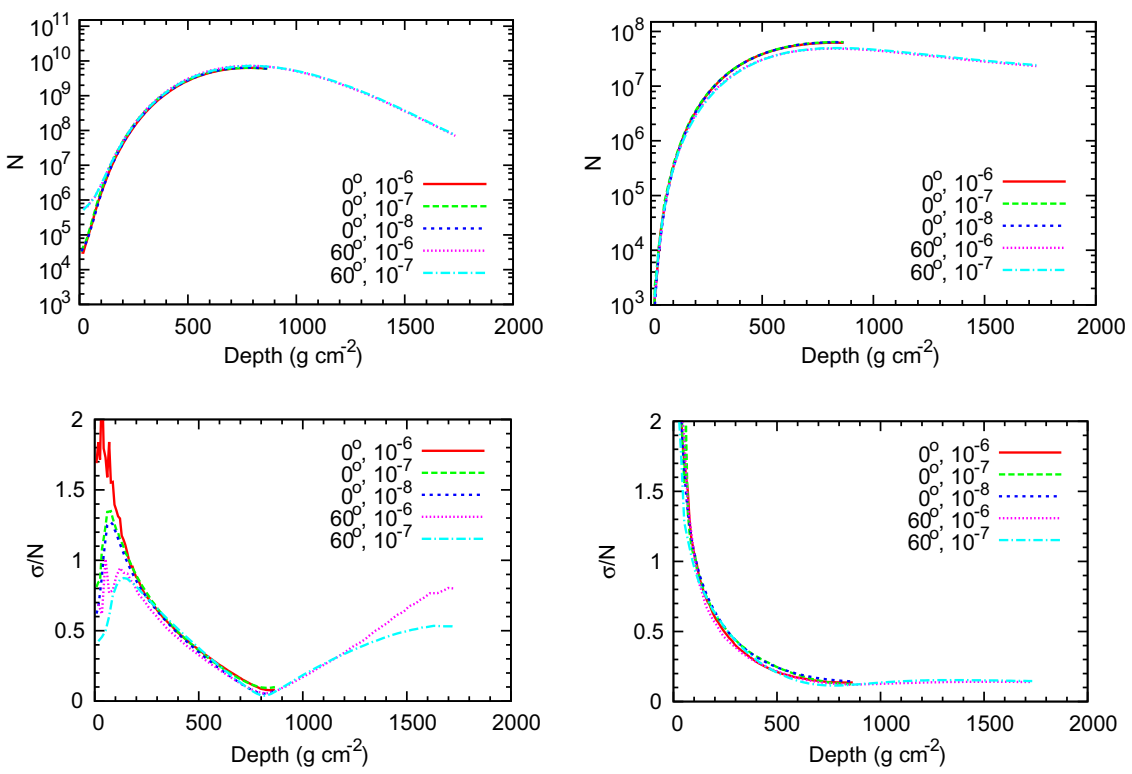

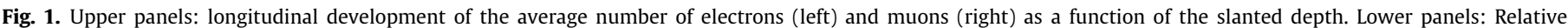

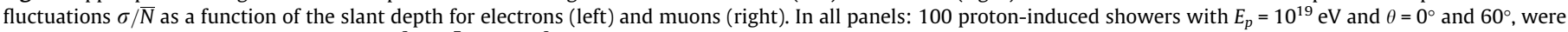
simulated with relative thinning $R_{\text {thin }}=10^{-6}, 10^{-7}$ and $10^{-8}$. 

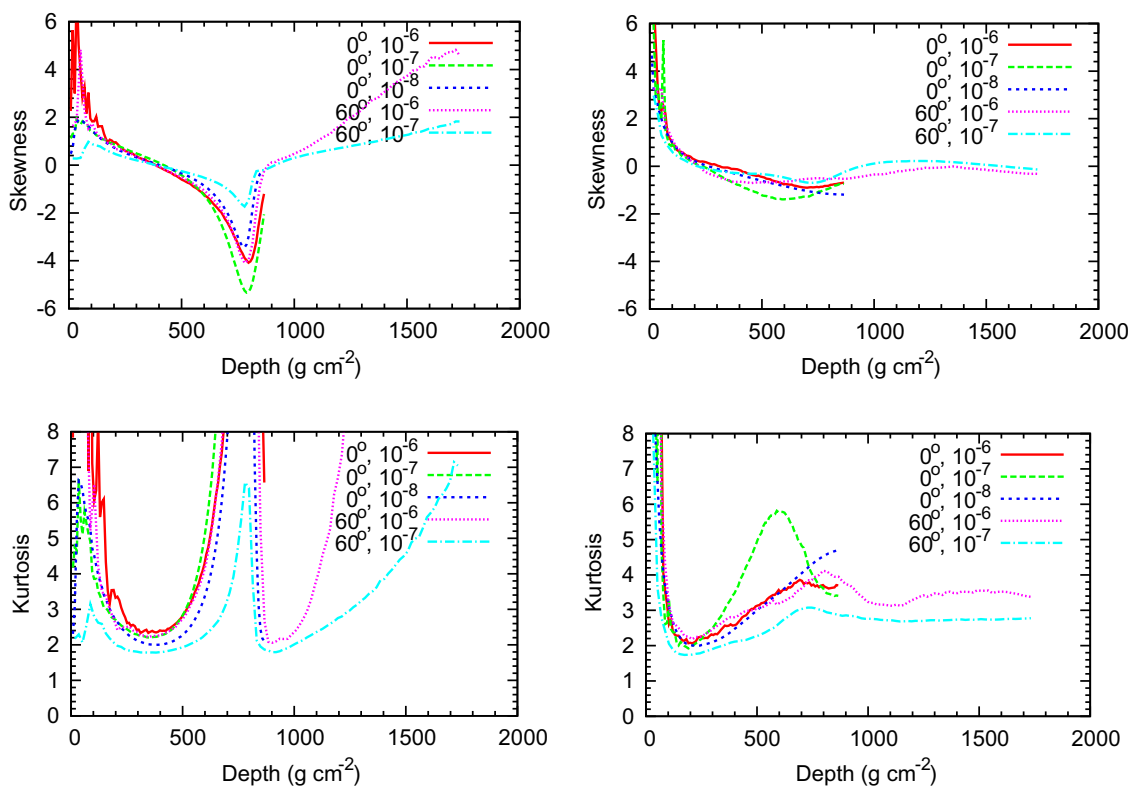

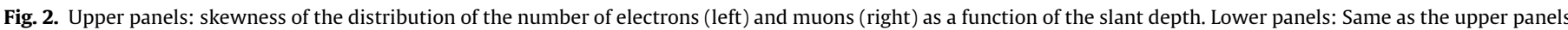
for the kurtosis. In all panels: 100 proton-induced showers of $E_{p}=10^{19} \mathrm{eV}$ with $\theta=0^{\circ}$ and $60^{\circ}$ were simulated with relative thinning $R_{\text {thin }}=10^{-6}$, $10^{-7}$ and $10^{-8}$.
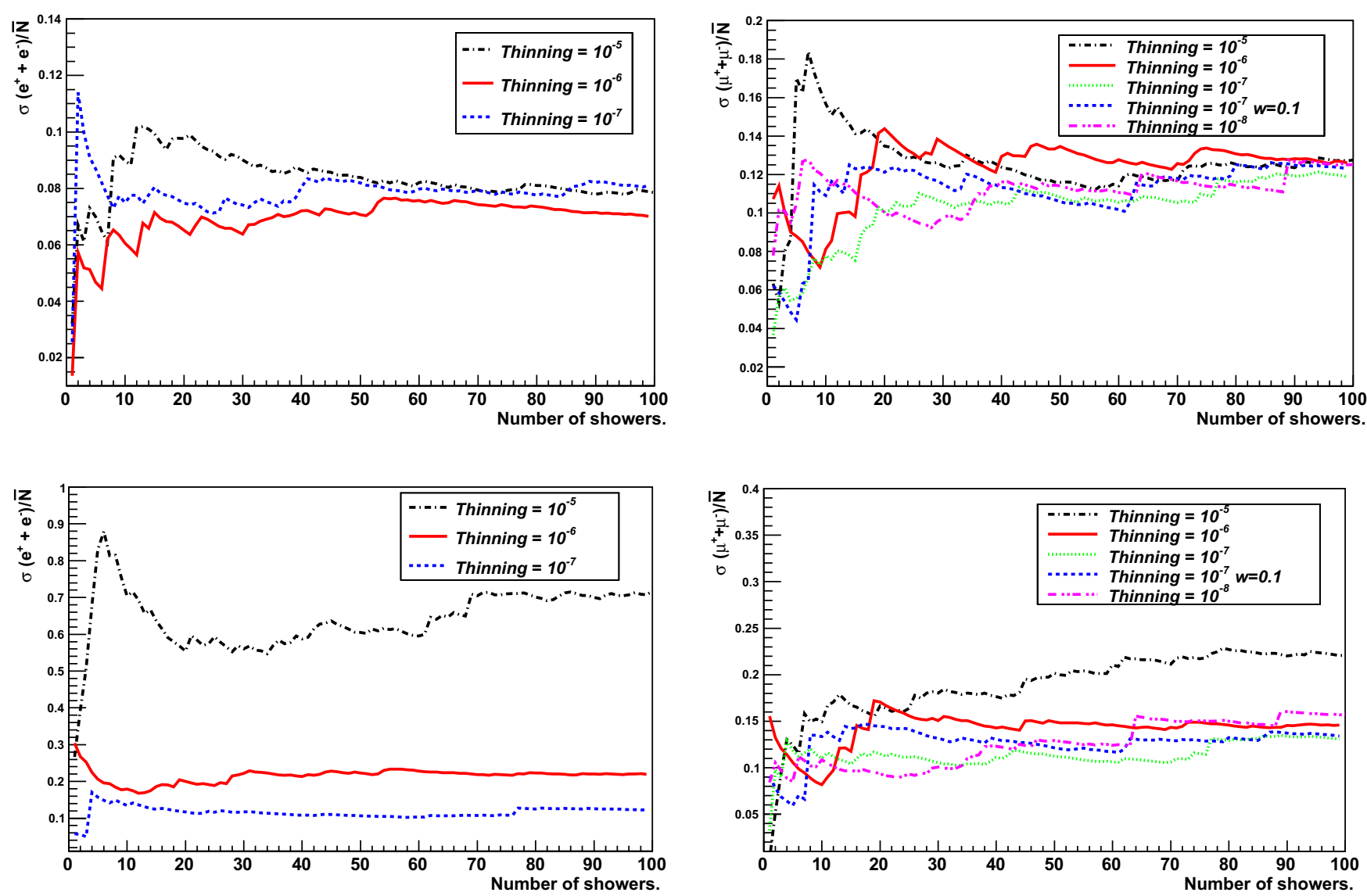

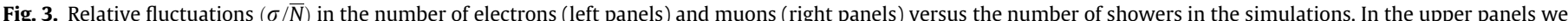

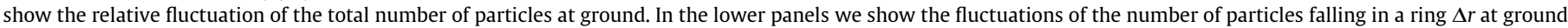

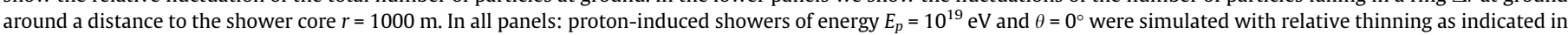
the insets.

in the whole ground. Also, the fluctuations in the ring have a stronger dependence with the thinning level used than those in the whole ground. This is easy to understand. An entry of weight $w$ falling in the ring represents $w$ particles, so that by losing or gaining 
just a single entry, one would lose or gain $w$ particles and the fluctuations are enlarged. This effect is not so strong when accounting for all the particles falling anywhere on the ground. In Fig. 3 it can also be clearly seen that no reliable evaluation of the fluctuations can be done with less than about 20 simulated showers, especially in the case of fluctuations in the ring. It can also be seen that a thinning level of $R_{\text {thin }}=10^{-6}$ or larger, introduces large artificial fluctuations, so that the shower to shower physical fluctuations cannot be evaluated reliably. This is however no obstacle to approximately estimate the physical shower to shower fluctuations as will be shown in the following.

\subsubsection{Physical shower to shower fluctuations at ground}

In the Appendix we prove that the distribution of the number of particles $N$ as obtained in thinned Monte Carlo simulations of extensive air showers has a mean $\bar{N}$ and a standard deviation $\sigma$ given by:

$\bar{N}=\bar{N}_{e} \bar{w}$,

and

$\sigma^{2}=\bar{N}_{e} \Omega^{2}+\bar{w}^{2} s^{2}$

where,

- $\bar{N}_{e}$ and $s$ are respectively the mean and the standard deviation of the distribution of the number of entries $N_{e}$ falling in a given ring around shower axis in each shower, i.e., the distribution of the number of non-thinned (explicitly sampled) particles in the simulation. The standard deviation of $N_{e}$ is simply the RMS of the distribution of $N_{e}$ over many showers.

- $\bar{w}$ and $\Omega$ are respectively the mean and the standard deviation of the distribution of weights assigned to the entries. The standard deviation of $w$ can be calculated from the RMS of the distribution of weights in a single shower (provided there are enough entries in that particular shower falling in the region of interest at ground), or from the RMS of the distribution of weights over many showers.

Of course Eq. (3) is exact since the thinning algorithm is designed to reproduce it. For Eq. (4), the proof only assumes that the probability for an entry to have a given weight $w$ is independent of the probability of a shower to have a given number of entries $N_{e}$. This is only approximate since the total number of entries and their weights are constrained by energy conservation.

One can interpret Eq. (4) as follows. If all entries (sampled particles) had the same weight equal to $\bar{w}$ in all the simulated showers, then the distribution of weights would not fluctuate from shower to shower and we would get $\Omega=0$. In this limit we would obviously have $\sigma=\bar{w} s$. This special case of Eq. (4) was also found in [14]. In the particular case in which all weights are equal to $\bar{w}=1$, i.e. the shower is fully simulated and the thinning procedure is not applied, then clearly $N=N_{e}, \sigma=s$ and the fluctuations would be obviously dominated by the true, physical shower to shower fluctuations. In the opposite limit, if we imagine that showers always have the same number of entries equal to $\bar{N}_{e}$, i.e. there are no physical shower to shower fluctuations, then we would get $s=0$, and the fluctuation in the number of particles would be solely due to the fluctuations of the weight of the entries, i.e., the fluctuations would be dominated by the thinning procedure and $\sigma^{2}=\bar{N}_{e} \Omega^{2}$. Clearly in these limits the first term of Eq. (4) can be identified with the artificial fluctuations introduced by the thinning procedure $\left(\sigma_{\text {thin }}^{2}=\bar{N}_{e} \Omega^{2}\right)$, and the second term with the true (physical) shower to shower fluctuations $\left(\sigma_{\text {phys }}^{2}=\bar{w}^{2} s^{2}\right)$.

In the following, and by means of our Monte Carlo simulations, we numerically study to which extent the two terms in Eq. (4) allow us to split the shower-to-shower fluctuations into artificial and true ones (as the limiting cases suggest), and hence allow us to estimate the effect of thinning in a particular set of simulations. This will clearly depend on the thinning level as shown below. Before that, we numerically demonstrate that Eq. (4) accounts for all the fluctuations (artificial and physical) appearing in simulations of EAS with thinning.

To see this in detail, we have calculated in our Monte Carlo simulations the average weight $\bar{w}$ and the sigma of the distribution of weights $\Omega$, the average number of entries $\bar{N}_{e}$ and the corresponding sigma of its distribution $s$, as well as the average number of particles $\bar{N}$ and the sigma of its distribution $\sigma$, both for electrons and muons, and compared them to what is predicted by Eq. (4).

Firstly, we have verified that the average number $\bar{N}$ of electrons and muons at different distances to the shower axis $r$ are rather independent of the thinning level, as expected since the thinning algorithm is designed to reproduce the average values of the numbers of particles in the shower. This is however not the case for the relative fluctuations $\sigma / \bar{N}$ shown in Fig. 4, which depend strongly on the thinning level used in the simulations. For both electron and muons $\sigma / \bar{N}$ has a minimum at the distance at which the number of particles is largest. Also, as expected, the fluctuations can be seen to converge to a common value at each distance as the thinning level decreases, because the effect of thinning is increasingly less important, fluctuations are increasingly dominated by the physical shower to shower fluctuations. The artificial fluctuations introduced by thinning in the case of electrons, do not contribute equally to the total fluctuation at all distances from the core as expected. It can be seen for instance that the relative fluctuation
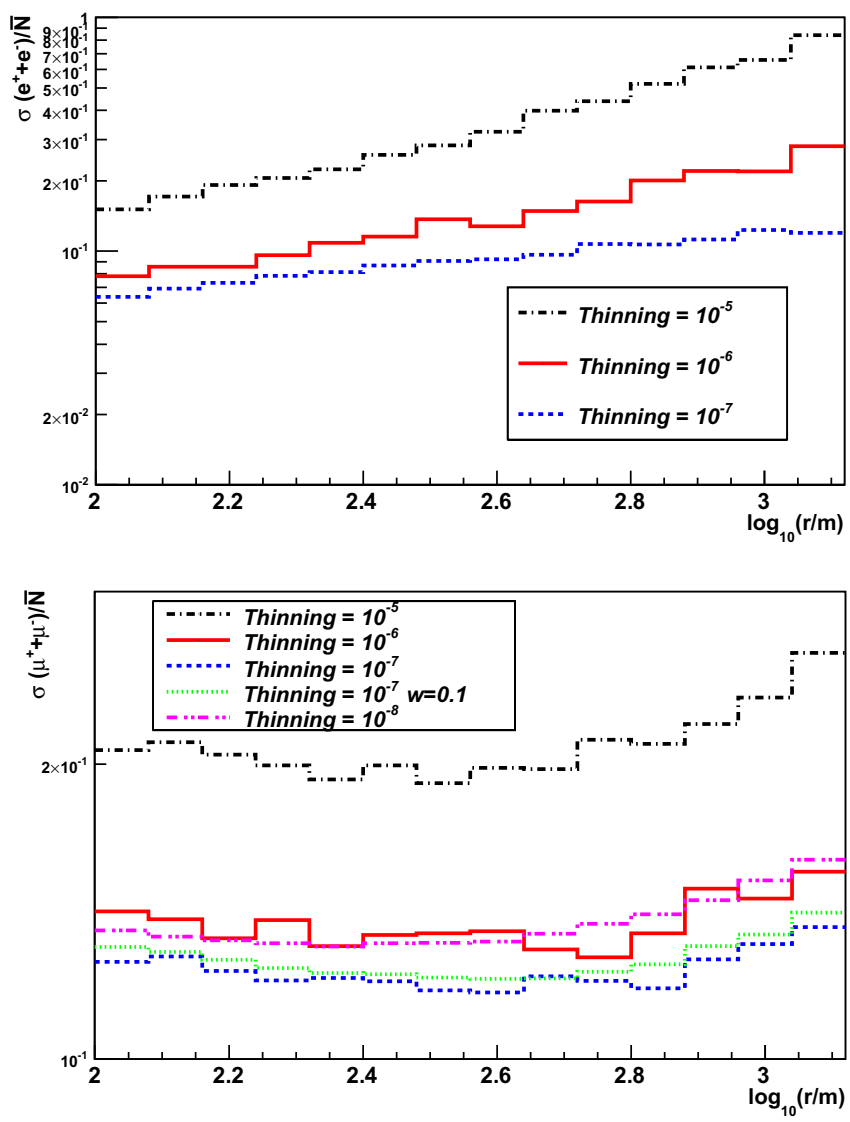

Fig. 4. The relative fluctuation $\sigma / \bar{N}$ in the number of electrons (upper panel) and muons (lower panel) at ground, as a function of the logarithm of the distance to the shower core. 100 proton-showers of energy $E_{p}=10^{19} \mathrm{eV}$ and $\theta=0^{\circ}$ were simulated with different thinning levels as indicated in the insets. 

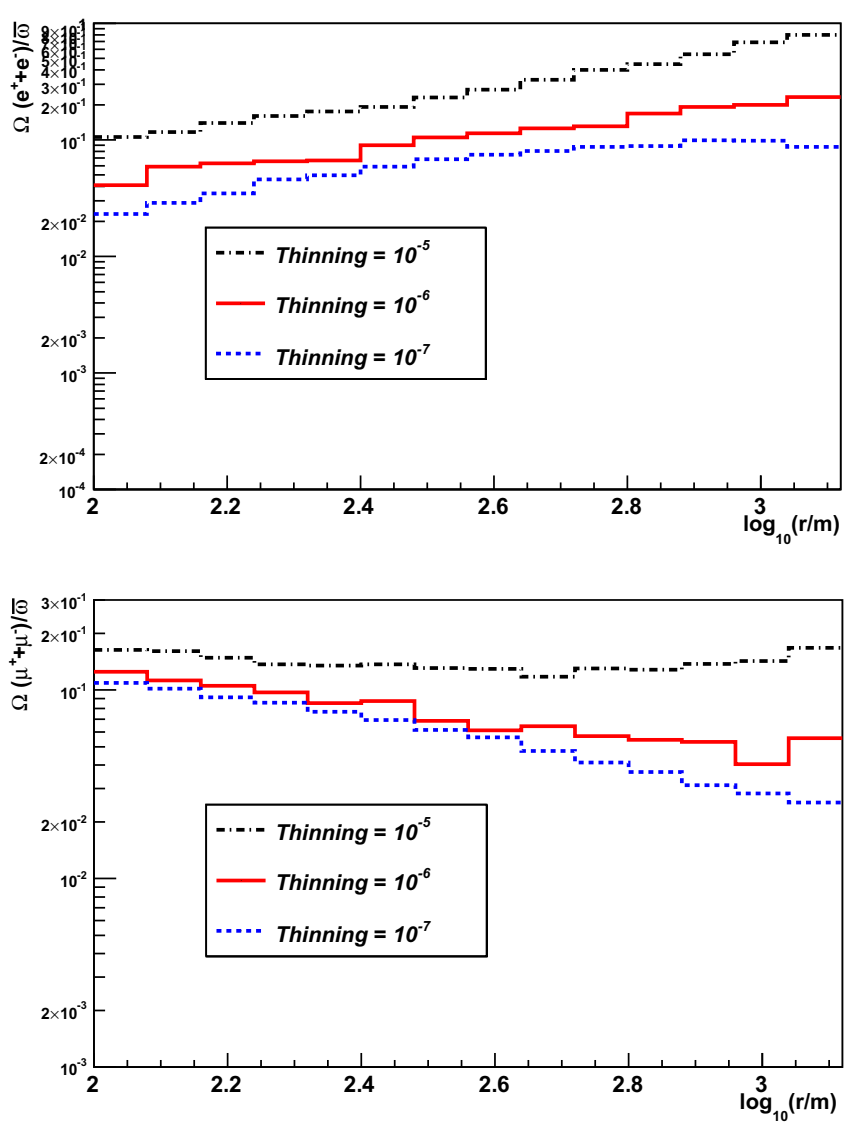

Fig. 5. Relative fluctuations of the distribution of weights $(\Omega / \bar{w})$ of electrons (upper panel) and muons (lower panel) at ground as a function of the logarithm of the distance to the core, for the same sets of shower simulations as in Fig. 4.

rises with $r$ but the increase gets smaller for the smallest thinning levels used in this work.

From our simulations we also obtain, as expected, that the average weight $\bar{w}$ assigned in the process of thinning to electrons and muons is simply proportional to the thinning level $\bar{w} \propto R_{\text {thin }}$. In Fig. 5 we plot the relative fluctuations of the distribution of weights $\Omega / \bar{w}$ for electrons, and muons as a function of distance to the shower core. As expected the fluctuation of the weight decreases as $R_{\text {thin }}$ decreases and the showers are less thinned. Also, for values of $R_{\text {thin }}<10^{-5}$ the relative fluctuation $\Omega / \bar{w}$ is roughly independent of the thinning level, and as a consequence we have that approximately $\Omega \propto R_{\text {thin }}$.

Regarding the average number of entries $\bar{N}_{e}$, we have verified in the simulations that $\bar{N}_{e} \propto R_{\text {thin }}^{-1}$, as imposed by the constraint in Eq. (3) that the average number of particles $\bar{N}$ should be independent of $R_{\text {thin }}$, together with the fact that $\bar{w} \propto R_{\text {thin }}$. In Fig. 6, we show the relative fluctuation in the number of entries $s / \bar{N}_{e}$. For small thinning levels $\left(R_{\text {thin }}<10^{-6}\right)$, we find that the relative fluctuation is approximately independent of the thinning level, implying that $S \propto R_{\text {thin }}^{-1}$.

Finally, in Fig. 7, we compare the relative fluctuation of the number of particles $\sigma / \bar{N}$ obtained directly in Monte Carlo simulations, with that predicted by Eq. (4), using the values of $\bar{w}, \Omega, \bar{N}_{e}$ and $s$ obtained in the same simulations. The comparison is shown for thinning levels $R_{\text {thin }}=10^{-5}, 10^{-6}$ and $10^{-7}$. The agreement between the $\sigma / \bar{N}$ obtained in Monte Carlo simulations, and that predicted by Eq. (4) is at the level of $<20 \%$ for electrons and $<5 \%$ for muons, confirming that Eq. (4) accounts for all the fluctuations (artificial and physical) appearing in the simulations of EAS with thinning.
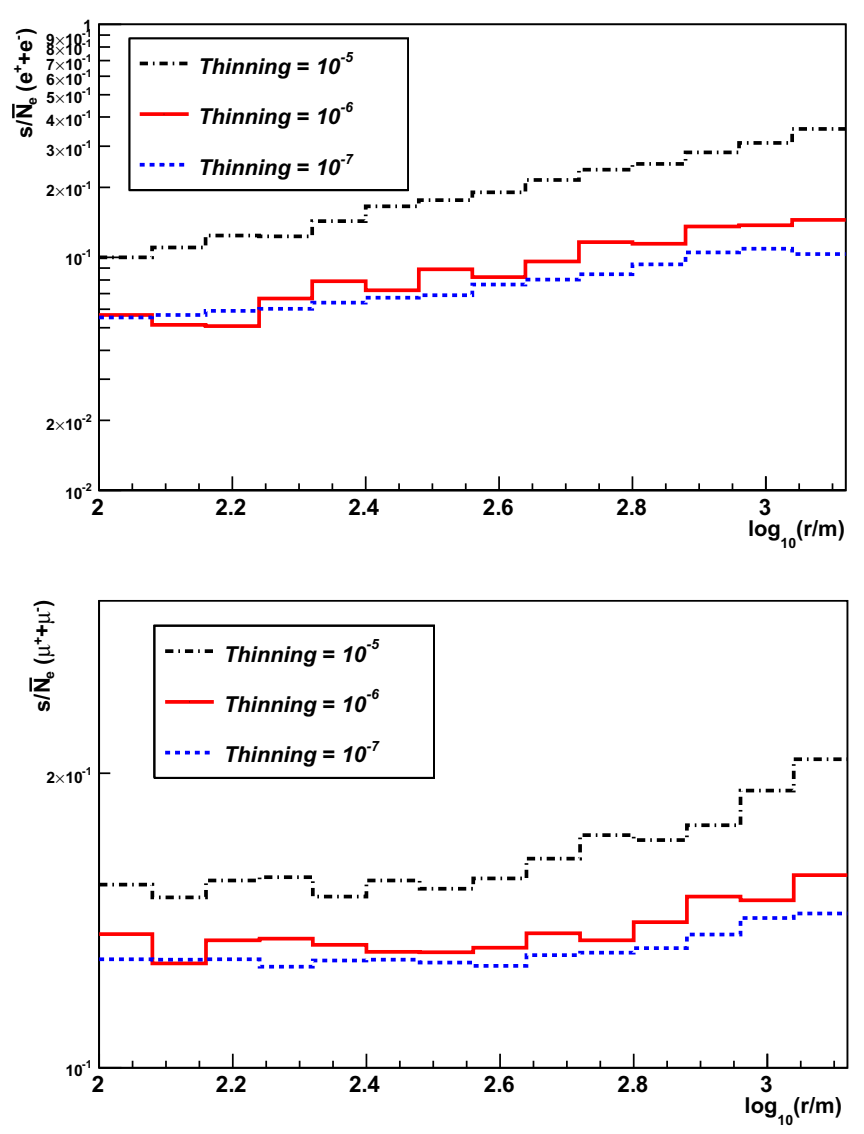

Fig. 6. Relative fluctuation $\left(s / \bar{N}_{e}\right)$ of the distribution of the number of entries for electrons (upper panel) and muons (lower panel) at ground, as a function of the logarithm of the distance to the core, for the same sets of shower simulations as in Fig. 4.

In Fig. 8 we plot the two terms in Eq. (4) as a function of the distance to the shower core for different thinning levels. For thinning level $R_{\text {thin }} \sim 10^{-7}$ (and smaller) the artificial fluctuations introduced by thinning are very small, and we approach the limiting case explained above in which the identification of the second term of Eq. (4) with the physical fluctuations is exact. For intermediate thinning levels $R_{\text {thin }} \sim 10^{-6}$ the artificial fluctuations are a factor of 3 larger than those at $R_{\text {thin }}=10^{-7}$, however the second term of Eq. (4) predicts that the fluctuations $\sigma_{\text {phys }}$ are very similar to those obtained with smaller thinning levels, despite the fact that the artificial fluctuations are not negligible - as a matter of fact they are of the same order of the physical fluctuations. This gives support to our identification of $\sigma_{\text {phys }}$ with the true physical fluctuations even for a relatively large thinning level such as $10^{-6}$ which is commonly used in shower simulations. Finally, for thinning levels $R_{\text {thin }} \sim 10^{-5}$ or larger, artificial fluctuations dominate, the physical fluctuations are almost completely erased, and the second term in Eq. (4) cannot reproduce them.

From the scalings with $R_{\text {thin }}$ of the different magnitudes involved in Eq. (4) obtained before, it is straightforward to deduce that $\sigma_{\text {thin }} \propto R_{\text {thin }}$, while $\sigma_{\text {phys }}$ should be approximately independent of $R_{\text {thin }}$ for thinning levels $10^{-6}$ and smaller. This is also seen in Fig.8.

To further check the conclusions above, we have run full simulations (i.e. with no thinning) of air showers and we have compared the shower-to-shower fluctuations obtained in this case - which are solely due to the physics fluctuations since the thinning procedure is not applied - with the fluctuations predicted by the second term of Eq. (4) in simulations with different thinning levels. Given 

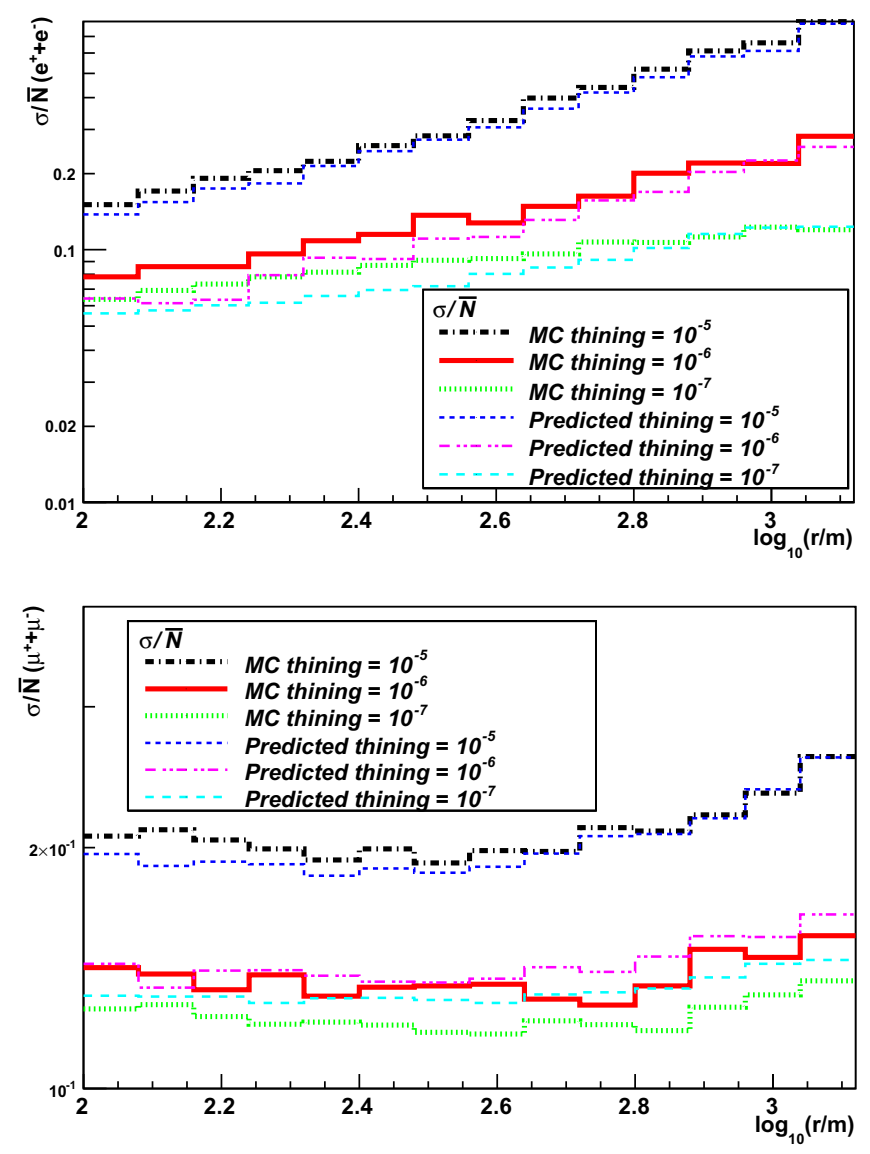

Fig. 7. Relative fluctuations $\sigma / \bar{N}$ of the distribution of number of electrons (upper panel) and muons (lower panel) at ground, as a function of the logarithm of the distance to the core, for the same sets of shower simulations as in Fig. 4. The $\sigma / \bar{N}$ obtained in the simulations (MC) is compared to that predicted by Eq. (4).

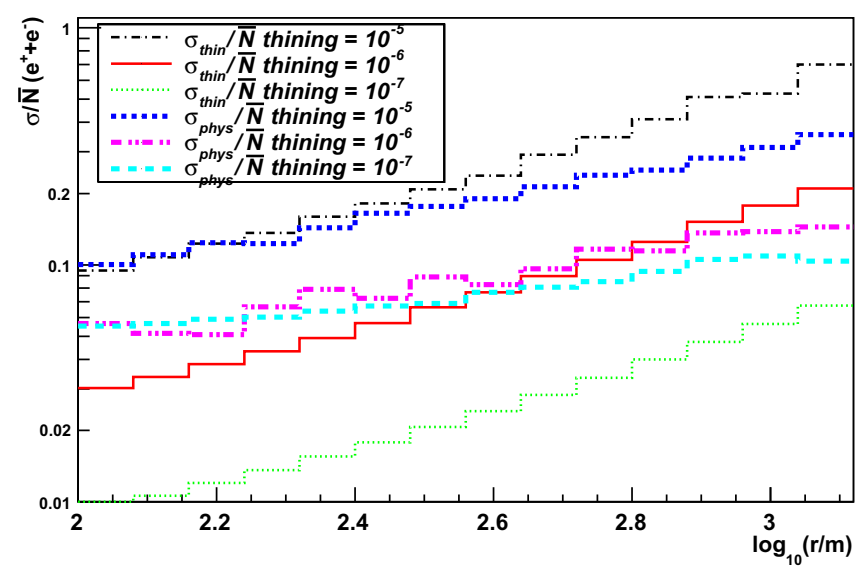

Fig. 8. Relative physical $\sigma_{\text {phys }} / \bar{N}$ and thinning fluctuations $\sigma_{\text {thin }} / \bar{N}$ (as predicted by Eq. (4)) of the distribution of number of electrons at ground, as a function of the logarithm of the distance to the core, for the same sets of shower simulations as in Fig. 4.

that it is impractical to run full simulations at an energy of $10^{19} \mathrm{eV}$, we have instead run 100 proton showers at lower energies, namely $10^{15} \mathrm{eV}$. Our results are shown in Fig. 9 where it can be seen that the physics fluctuations predicted by the second term of Eq. (4) reproduce the fluctuations in showers with no thinning - at the $25 \%$ level or better - for thinning levels $\sim 10^{-6}$ and smaller, in agreement with our previous conclusions.

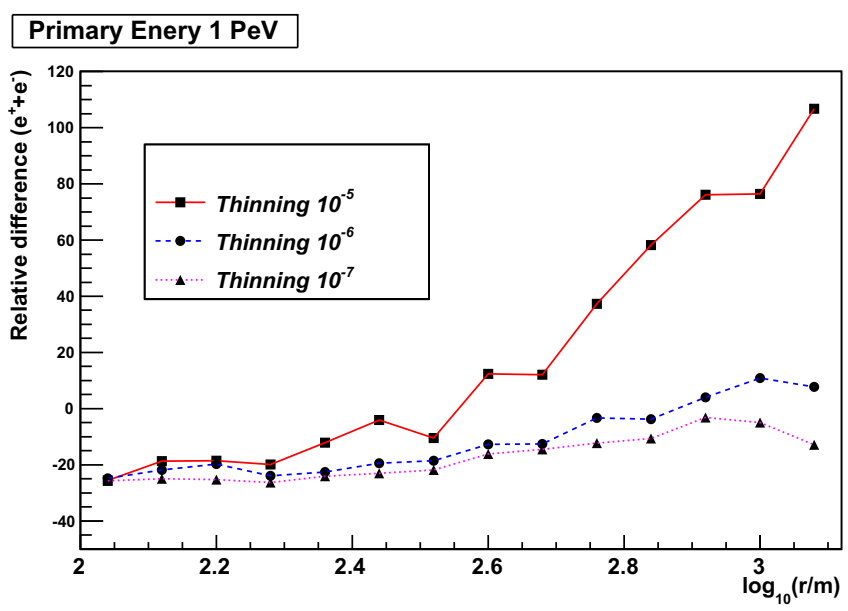

Fig. 9. Relative difference between the physical fluctuations in 100 proton showers of primary energy $E_{p}=10^{15} \mathrm{eV}$ and zenith angle $\theta=0^{\circ}$ simulated without applying the thinning algorithm, and the physical fluctuations predicted by the second term of Eq. (4) in simulations with different thinning levels (see inset). The relative difference calculated in percentage as $\sigma_{\text {phys }}$ (with thinning) $-\sigma_{\text {phys }}$ (no thinning) normalized to the $\sigma_{\text {phys }}$ (no thinning) is shown as a function of the distance to the shower axis.

After having shown that the second term in Eq. (4) reproduces the physical shower-to-shower fluctuations for thinning levels $R_{\text {thin }} \sim 10^{-6}$ and smaller, in the next two sections we study the behaviour of physical fluctuations.

\section{Dependence of fluctuations at ground on the number of particles}

Let us now consider the dependence of fluctuations on the size of the ring around a distance to the shower core $r$, where particles are collected in the simulation. Let us first consider how the density of particles is evaluated. If $\bar{N}(r, \Delta r)$ is the average number of particles at distance $r$ in a small bin $\Delta r$ (here we assume cylindrical symmetry around the shower axis, but the argument does not depend on this simplification), then the density of particles $\rho(r)$ can be defined as:

$\rho(r)=\lim _{\Delta r \rightarrow 0} \frac{\bar{N}(r, \Delta r)}{2 \pi r \Delta r}$.

In the limit $\Delta r \rightarrow 0, \rho(r)$ is finite, at least for $r \neq 0$. However, the same is not true for the fluctuations, so that in general one can not define a "density of fluctuations" $\rho_{\sigma}(r)$. We can see this in a simple example. Assume that $\sigma(r, \Delta r)$ is the standard deviation of the distribution of the number of particles in a bin of size $\Delta r$ and at a distance $r$. One could try to define the density of fluctuations as

$\rho_{\sigma}(r)=\lim _{\Delta r \rightarrow 0} \frac{\sigma(r, \Delta r)}{2 \pi r \Delta r}$.

If the fluctuations in the number of particles at ground were purely Poissonian, we would have

$\sigma(r, \Delta r)=\sqrt{N(r, \Delta r)}$,

and therefore,

$$
\begin{aligned}
\rho_{\sigma}(r) & =\lim _{\Delta r \rightarrow 0} \frac{\sqrt{N(r, \Delta r)}}{2 \pi r \Delta r}=\lim _{\Delta r \rightarrow 0} \frac{\sqrt{2 \pi r \Delta r \rho(r)}}{2 \pi r \Delta r} \\
& =\lim _{\Delta r \rightarrow 0} \frac{\sqrt{\rho(r)}}{\sqrt{2 \pi r \Delta r}} \rightarrow \infty,
\end{aligned}
$$

i.e., we can not define a density of fluctuations for Poissonian shower to shower fluctuations in the number of particles. The 
dependence on bin-size has been identified with the fractal structure of showers [15]. In the case of Poissonian fluctuations in the number of particles, the deduced behaviour is $\rho_{\sigma}(r) \propto \Delta r^{\alpha}$ with $\alpha=-1 / 2$, and we would be tempted to identify the coefficient $\alpha$ with a fractal exponent. However notice that for a Poissonian process no fractal structure is implied at all and it would be erroneous to call it a fractal exponent.

On the other hand, in the case in which the fluctuations behave as:

$\sigma(r, \Delta r)=f(r) \Delta r+O\left(\Delta r^{2}\right)$

where $f(r)$ is a function that does not depend on $\Delta r$, one can define a density of fluctuations as can be shown trivially applying Eq. (6). Remarkably, this is precisely the case of Furry's fluctuations [16], in which $\sigma \sim N=2 \pi r \rho(r) \Delta r$ and then $\rho_{\sigma}$ would be independent of $\Delta r$, i.e. $\alpha=0$. Recall that Furry statistics appears as a extremely simplified model of shower fluctuations [16], but it does take into account the branching structure of the shower (and therefore has an implicit fractal structure included).

To our knowledge, the actual behaviour of the fluctuations of showers and its dependence with the bin size is an open theoretical problem, with the theoretical prejudice ranging between those two extremes: purely Poissonian fluctuations $\sigma \sim \sqrt{N}$, and Furry fluctuations $\sigma \sim N$. For instance, for the longitudinal development of showers one can show that in fact both types of behaviour occur [17], i.e.,

$\sigma^{2}=a N^{2}+b N$
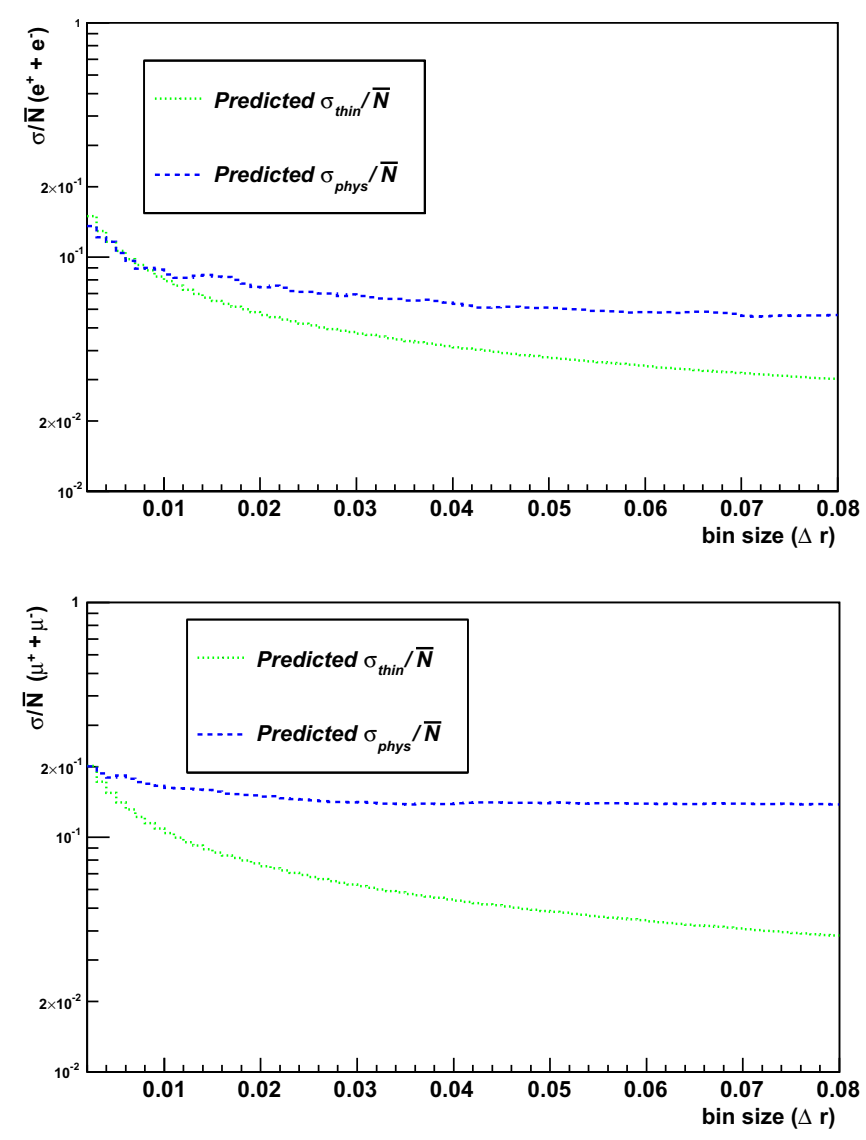

Fig. 10. Relative fluctuations $\sigma / \bar{N}$ of the distribution of the number of electrons (upper panel) and muons (lower panel) in a ring of width $\Delta r$, centered at $r=100 \mathrm{~m}$ at ground, as a function of the logarithm of size of the bin $\Delta r$. The simulations were performed with relative thinning level $R_{\text {thin }}=10^{-6}$. The two terms in Eq. (4) corresponding to fluctuations induced by thinning $\sigma_{\text {thin }}$ and physical fluctuations $\sigma_{\text {phys }}$ are also shown, see insets. where $a$ and $b$ vary slowly with primary energy [17]. Near the maximum of the shower, the first term dominates and fluctuations are not Poissonian. For the lateral distribution no such result exists but one would expect a similar conclusion.

In Fig. 10 we show the relative fluctuations in the number of particles as predicted by the two terms in Eq. (4) for thinning level $R_{\text {thin }}=10^{-6}$ for which we have shown before that the two terms in Eq. (4) predict the physical and artificial fluctuations. It can be seen that $\sigma_{\text {phys }} / \bar{N}$ is consistent with being flat with $\Delta r$, while $\sigma_{\text {thin }} / \bar{N}$ behaves as a power law (a fit gives $\sigma_{\text {thin }} / \bar{N} \propto \Delta r^{-1 / 2}$ ). These results suggest that the artificial fluctuations are Poissonian, while physical fluctuations behave as $\sigma_{\text {phys }} \propto N$.

\section{Dependence of shower to shower fluctuations on composition and depth of first interaction}

In this Section we study the influence of the fluctuations in the depth of first interaction on the overall shower to shower fluctuations of the number of particles. In Fig. 11 we plot the relative fluctuations $\sigma / \bar{N}$ in $10^{19} \mathrm{eV}$ proton and iron-induced showers. In all panels we show the results of our regular simulations, together with the results of a special set of simulations performed by fixing the depth of first interaction of the primary particle (proton or
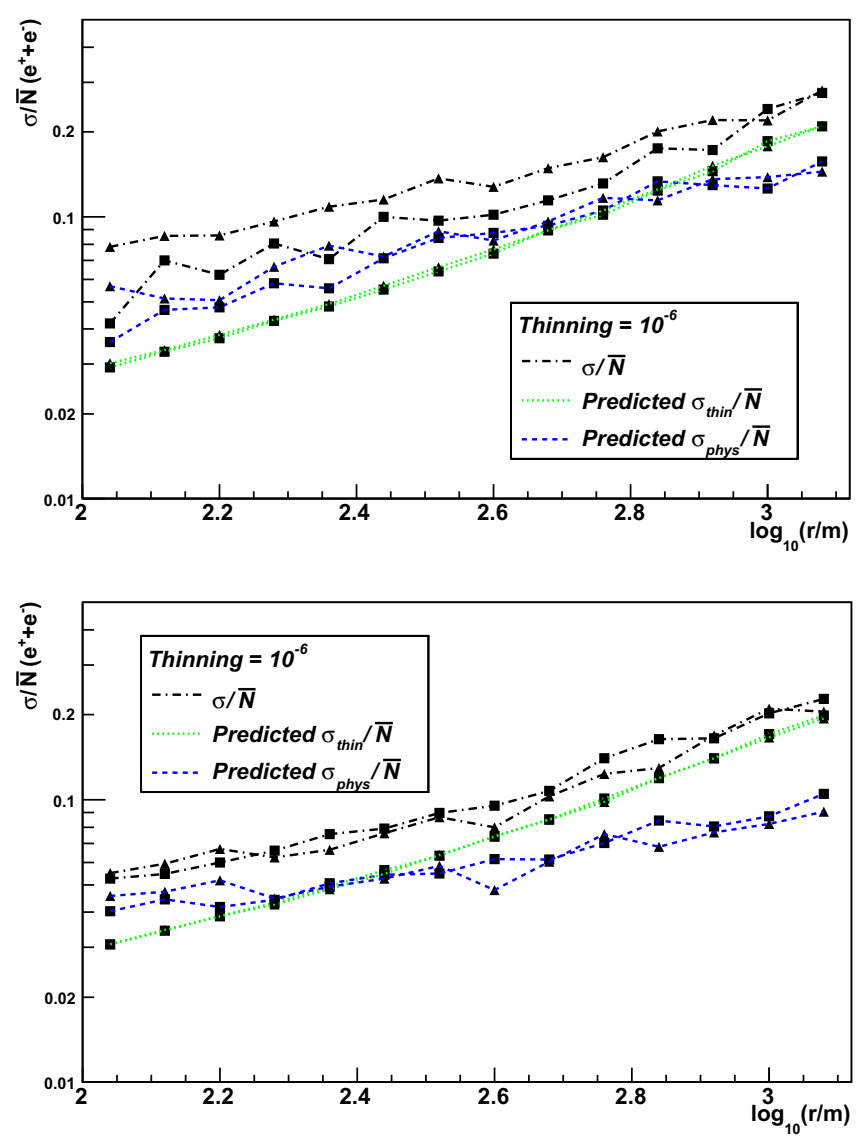

Fig. 11. Relative fluctuations $\sigma / \bar{N}$ of the distribution of number of electrons at ground, as a function of the logarithm of the distance to the core, for $10^{19} \mathrm{eV}$ proton showers (upper panel) and iron showers (lower panel) with $\theta=0^{\circ}$, and relative thinning level $R_{\text {thin }}=10^{-6}$. We show (squares) the result of fixing the first interaction depth at $44.9 \mathrm{~g} / \mathrm{cm}^{2}$ (mean interaction depth of $10^{19} \mathrm{eV}$ proton-air collisions predicted by the QGSJET01 model - upper panel) or $10.7 \mathrm{~g} / \mathrm{cm}^{2}$ (corresponding to the mean interaction depth of $10^{19} \mathrm{eV}$ iron-air collisions predicted by the QGSJET01 model - lower panel), and also the case in which the depth of first interaction fluctuates (triangles). The two terms in Eq. (4) corresponding to fluctuations induced by thinning $\sigma_{\text {thin }}$ and physical fluctuations $\sigma_{\text {phys }}$ are also shown in all cases, see insets. 
iron) at the value of its mean interaction depth predicted by the QGSJET model (namely $44.9 \mathrm{~g} / \mathrm{cm}^{2}$ for proton at $10^{19} \mathrm{eV}$ and $10.7 \mathrm{~g} / \mathrm{cm}^{2}$ for iron at the same energy). In all cases we use Eq. (4) to split the fluctuations into artificial and physical fluctuations, and we also show them in the figures.

Firstly, it is interesting to see that the artificial fluctuations in the number of electrons in iron showers are approximately equal to those in proton showers, while the physical fluctuations are smaller in iron than in proton-induced showers. The latter observation is a well-known effect which is attributed to the fact that showers initiated by a nuclei can be considered, in a first approximation, as a superposition of $A$ (atomic mass) nucleons, each with an energy $E / A$ with $E$ the energy of the primary nucleus.

It is rather remarkable that the relative fluctuations in the number of particles $\sigma / \bar{N}$ in the two different sets of simulations (fixing or varying the depth of the first interaction point), are essentially the same. One could think that this is due to the fluctuations induced by thinning which mask the effect of the fluctuations of the depth of first interaction, however this does not seem to be the case, since as can be seen in Fig. 11 neither the first term of Eq. (4) (the thinning fluctuations), nor the second term (the physical fluctuations) change much when varying or fixing the depth of first interaction. We conclude that the relative shower to shower fluctuations of the number of particles at ground $\sigma / \bar{N}$ are rather insensitive to the physical fluctuations of the depth of first interaction, and that these are of the same order as the fluctuations occurring in the subsequent secondary interactions in the shower. This is related to the fact that the maximum of a shower at $\theta=0^{\circ}$, where the fluctuations are minimum [13], occurs near the ground. For other zenith angles a small difference appears in the physical fluctuations of the simulations performed with fixed and fluctuated first interaction point.

\section{Conclusions}

In this work we have performed a comprehensive study of shower to shower fluctuations by means of Monte Carlo simulations of extensive air showers. An understanding of the shower to shower fluctuations will help to improve the interpretation of cosmic-ray data.

The determination of the true, physical shower to shower fluctuations is hampered by the thinning procedure necessary to simulate in a practical manner air showers at EeV energies and above. However, we have shown that the artificial fluctuations induced by thinning $\left(\sigma_{\text {thin }}\right)$ can be identified and splitted from the physical fluctuations ( $\sigma_{\text {phys }}$ ) with the aid of Eq. (4), provided thinning levels $R_{\text {thin }} \sim 10^{-6}$ or smaller are used in the simulations. It is important to remark that for $R_{\text {thin }}=10^{-6}$ the artificial fluctuations are not negligible, in fact they are of the same order as the physical fluctuations, and hence Eq. (4) is able to reproduce them for a thinning level commonly used in shower simulations. We have also shown that Eq. (4) accounts for all, true and artificial fluctuations appearing in the simulations for thinning levels $R_{\text {thin }}<10^{-5}$. Moreover, Eq. (4) reproduces the expectation that as the thinning level decreases $R_{\text {thin }} \rightarrow 0$, and showers are less thinned, then the artificial fluctuations decrease, the physical ones become dominant, and they do not depend on $R_{\text {thin }}$.

We have shown in Section 5 that the physical shower to shower fluctuations of the number of particles at ground behave proportionally to the number of particles $N$, while the artificial fluctuations are Poissonian, i.e., behave as $\sqrt{N}$.

We have also shown that the size of the relative fluctuations due to the depth at which the first interaction initiating the shower occurs, is smaller or of the same order as the fluctuations occurring in the subsequent secondary interactions in the shower.

\section{Acknowledgements}

We thank V. Canoa, G. Rodriguez-Fernandez, T. Tarutina, I. Valiño and E. Zas for discussions and comments. P.M.H. was supported by Juan de la Cierva grant. We thank Centro de Supercomputación de Galicia (CESGA) for computer resources. This work was made possible with support from the Ministerio de Ciencia e Innovación, Spain under grant FPA 2007-65114 and Consolider CPAN; and of ALFA-EC funds in the framework of the HELEN (High Energy Physics Latin-American-European Network) project. J. A-M also thanks Xunta de Galicia (INCITE09 206336 PR) for financial support.

\section{Appendix A}

In this Appendix, we calculate the probability distribution of the number of particles $N$ in a given bin of $r$ distance to shower core, or of energy making some simplifying assumptions. We want to calculate the probability distribution of particles, possibly in a given bin of $r$ or of energy. We will assume that the probability for an entry (a non-thinned particle) to have a weight $w_{i}$ is given by $P_{w}\left(w_{i}\right)$. In addition the number of entries, $N_{e}$ in a given shower is a random variable with probability distribution $P_{e}\left(N_{e}\right)$. Our main simplifying assumption is the following: we will assume that $P_{w}$ and $P_{e}$ are independent of each other. This assumption is only approximate, because in a shower simulated with thinning both the entries and the weight assigned to each particle are controlled by the branching of the shower and therefore they must be related. However, as we will see a posteriori, the approximation is good enough for our purposes here, and it serves to clarify the role of thinning.

Under this approximation we can write the probability $P(N)$ of having $N$ particles as:

$$
\begin{aligned}
P(N)= & \sum_{N_{e}} P_{e}\left(N_{e}\right)\left[\int d w _ { 1 } \cdots d w _ { N _ { e } } P _ { w } ( w _ { 1 } ) \cdots P _ { w } ( w _ { N _ { e } } ) \delta \left(w_{1}+\cdots\right.\right. \\
& \left.\left.+w_{N_{e}}-N\right)\right] .
\end{aligned}
$$

where the $\delta$-function expresses the constraint that the sum of weights is equal to the total number of particles.

In what follows we evaluate this expression first by making further assumptions about the shape of $P_{e}$ and $P_{w}$, and afterwards in the general case using the characteristic function, related to the probability distribution. The definitions of cumulants and of the characteristic function can be found in any text book on statistics, for instance [18].

We start with the integral

$$
\begin{aligned}
I\left(N_{e}, N\right)= & \int d w_{1} \cdots d w_{N_{e}} P_{w}\left(w_{1}\right) \cdots P_{w}\left(w_{N_{e}}\right) \delta\left(w_{1}+\cdots+w_{N_{e}}\right. \\
& -N),
\end{aligned}
$$

and introduce the Fourier representation for the delta function.

$$
I\left(N_{e}, N\right)=\int d w_{1} \cdots d w_{N_{e}} P_{w}\left(w_{1}\right) \cdots P_{w}\left(w_{N_{e}}\right) \frac{1}{2 \pi} \int d k e^{i k\left(w_{1}+\cdots+w_{N_{e}}-N\right)} .
$$

Changing the order of integration gives

$$
\begin{aligned}
I\left(N_{e}, N\right) & =\frac{1}{2 \pi} \int d k e^{-i k N} \int d w_{1} P_{w}\left(w_{1}\right) e^{i k w_{1}} \ldots \int d w_{N_{e}} P_{w}\left(w_{N_{e}}\right) e^{i k w_{N_{e}}} \\
& =\frac{1}{2 \pi} \int d k e^{-i k N}\left[\int d w P_{w}(w) e^{i k w}\right]^{N_{e}} .
\end{aligned}
$$

To further continue with the evaluation of $P(N)$ we need to make additional approximations. We assume that $P_{w}$ is a Gaussian distribution with average $\bar{w}$ and $\operatorname{rms} \Omega$

$P_{w}(w)=A e^{-(w-\bar{w})^{2} /\left(2 \Omega^{2}\right)}$, 
where $A=1 / \sqrt{2 \pi \Omega^{2}}$ is the probability normalization. Its Fourier transformation is given by

$\int d w e^{i k w} P_{w}(w)=e^{i k \bar{w}} e^{-k^{2} \Omega^{2} / 2}$

Therefore,

$I\left(N_{e}, N\right)=\frac{1}{2 \pi} \int d k e^{-i k\left(N-N_{e} \bar{w}\right)} e^{-N_{e} k^{2} \Omega^{2} / 2}$,

For large values of $\bar{N}_{e}$, the sum in Eq. (11) can be approximated by an integral

$P(N)=\sum_{N_{e}} P_{e}\left(N_{e}\right) I\left(N_{e}, N\right) \approx \int d N_{e} P\left(N_{e}\right) I\left(N_{e}, N\right)$.

If we assume that $P_{e}\left(N_{e}\right)$ is also a Gaussian with average $\bar{N}_{e}$ and standard deviation $s$ and inserting Eq. (17) in Eq. (18) gives

$P(N)=\frac{1}{2 \pi} \int d k e^{-i k N} \int d N_{e} \frac{1}{\sqrt{2 \pi s^{2}}} e^{-\left(N_{e}-\bar{N}_{e}\right)^{2} /\left(2 s^{2}\right)} e^{i k N_{e} \bar{w}} e^{-k^{2} N_{e} \Omega^{2} / 2}$.

The integral over $N_{e}$ can be done analytically

$P(N)=\frac{1}{2 \pi} \int d k e^{-i k N} \exp \left[i \bar{N}_{e} \bar{w} k-\frac{1}{2}\left(\bar{N}_{e} \Omega^{2}+s^{2} \bar{w}^{2}\right) k^{2}+O\left(k^{3}\right)\right]$.

where we neglect in the exponential powers of $k$ larger than two, after applying the saddle point approximation. We arrive at the final expression

$P(N)=\frac{1}{\sqrt{2 \pi \sigma^{2}}} e^{-(N-\bar{N})^{2} /\left(2 \sigma^{2}\right)}$,

where

$\bar{N}=\bar{N}_{e} \bar{w}$,

$\sigma^{2}=\bar{N}_{e} \Omega^{2}+\bar{w}^{2} s^{2}$

Notice that the above expressions have the correct asymptotic behaviour. If the particles have no weight, $\bar{w} \rightarrow 1$ and $\Omega \rightarrow 0$, then the average number of particles is equal to the average number of entries (non-thinned particles) $\bar{N}=\bar{N}_{e}$ and $\sigma=s$. On the extreme case of a strongly thinned shower in which all particles are grouped together in a single entry $\left(\bar{N}_{e}=1, s=0\right)$ then $\bar{N}=\bar{w}$ and $\sigma=\Omega$, as expected.

The above result is general and valid for any probability distribution for $P_{w}$ and $P_{e}$. The only requirement is the "factorization" property given in Eq. (11). From Eq. (14), we introduce the characteristic function for the probability distribution $P_{w}$,

$\widetilde{P}_{w}(k)=\int d w P_{w}(w) e^{i k w}$,

which, in general can be written as:

$\widetilde{P}_{w}(k)=\exp \left[i k a_{1}-\frac{1}{2} a_{2} k^{2}+\cdots\right]=e^{i g(k)}$, where the coefficients of the expansion of $g(k)$ are related to the cumulants of the distribution of $P_{w}$. For instance $a_{1}=\bar{w}, a_{2}=\Omega^{2}$, etc. Then Eq. (11) reads

$P(N)=\frac{1}{2 \pi} \int d k e^{-i k N} \int d N_{e} P_{e}\left(N_{e}\right) e^{i N_{e} g(k)}$.

We define

$\widetilde{P}_{e}(q)=\int d N_{e} P_{e}\left(N_{e}\right) e^{i q N_{e}}=\exp \left[i b_{1} q-\frac{1}{2} b_{2} q^{2}+\cdots\right]$,

where as before $b_{1}=\bar{N}_{e}$ and $b_{2}=s^{2}$. Then we get

$$
\begin{aligned}
P(N) & =\frac{1}{2 \pi} \int d k e^{-i k N} \widetilde{P}_{e}(g(k)) \\
& =\frac{1}{2 \pi} \int d k e^{-i k N} \exp \left[i b_{1} g(k)-\frac{1}{2} b_{2} g(k)^{2}+\cdots\right],
\end{aligned}
$$

where the function $g(k)=k \bar{w}+i / 2 k^{2} \Omega^{2}+\cdots$. Then after some algebra

$P(N)=\frac{1}{2 \pi} \int d k e^{-i k N} \exp \left[i k \bar{w} \bar{N}_{e}-\frac{1}{2} k^{2}\left(s^{2} \bar{w}^{2}+\bar{N}_{e} \Omega^{2}\right)+\cdots\right]$

The coefficients of the expansion around $k=0$ are again the cumulants of the distribution $P(N)$, therefore we simply read the result given above in Eq. (22). But, as a bonus, we obtain also all the other cumulants. For instance for the skewness we obtain

$\gamma_{3}=\frac{M_{3}}{\sigma^{3}}=\frac{1}{\sigma^{3}} \times\left(m_{3} \bar{N}_{e}+3 \bar{w} s^{2} \Omega^{2}+\bar{w}^{3} M_{e}\right)$

where $m_{3}$ is the third central moment $\left.\left(m_{3}=\left\langle(w-\bar{w})^{3}\right)\right\rangle\right)$ of the weight distribution and $M_{e}$ is the third central moment of the distribution of the number of entries. In the same way, one can easily obtain other cumulants from the above expressions.

\section{References}

[1] M. Nagano, A. Watson, Rev. Mod. Phys. 72 (2000) 689.

[2] C.A. García Canal et al., Phys. Rev. D 79 (2009) 054006.

[3] A.M. Hillas, Proc of the Paris Workshop on Cascade simulations, in: J. Linsley, A.M. Hillas (Eds.), 1981, pp. 39.;

A.M. Hillas, Nucl. Phys. B (Proc. Suppl.) 52B (1997) 29.

[4] V.A. Kuzmin, G.I. Rubtsov, JETP Lett. 85 (2007) 535.

[5] D.S. Gorbunov, G.I. Rubtsov, S.V. Troitsky, Phys. Rev. D 76 (2007) 043004

[6] S.J. Sciutto, Proc. 27th ICRC (Hamburg) 1 (2001) 237.

[7] S.J. Sciutto, AIRES User's Manual and Reference Guide; version 2.6.0, 2002. $<$ www.fisica.unlp.edu.ar/auger/aires>.

[8] N.N. Kalmykov, S.S. Ostapchenko, Yad. Fiz. 56 (1993) 105;

N.N. Kalmykov, S.S. Ostapchenko, Phys. At. Nucl. 56 (1993) 346;

N.N. Kalmykov, S.S. Ostapchenko, A.I. Pavlov, Bull. Russ. Acad. Sci. (Physics) 58 (1994) 1966.

[9] M. Kobal et al., Pierre Auger collaboration, Astropart. Phys. 15 (2001) 259.

[10] F. Schmidt, M. Ave, L. Cazon, A.S. Chou, Astropart. Phys. 29 (2008) 355.

[11] P. Billoir, Astropart. Phys. 30 (2008) 270.

[12] M. Ave et al., Nucl. Instrs. Methods Phys. Res. A 578 (2007) 180.

[13] T.K. Gaisser, Cosmic Rays and Particle Physics, Cambridge Univ. Press, 1992.

[14] M. Risse, et al., Proc. of 27th ICRC, Hamburg, Germany, 2001, pp. 522.

[15] J. Kempa, M. Samorski, J. Phys. G: Nucl. Part. Phys. 24 (1998) 1039.

[16] W.H. Furry, Phys. Rev. 52 (1937) 569.

[17] R.A. Vazquez, Astropart. Phys. 6 (1997) 411.

[18] See for instance W.T. Eadie et al., Statistical Methods in Experimental Physics, North-Holland, Pub., Amsterdam, 1971. 